\title{
APSS-ASJ Best Clinical Research Award: Predictability of Curve Progression in Adolescent Idiopathic Scoliosis Using the Distal Radius and Ulna Classification
}

\author{
Jason Pui Yin Cheung, Prudence Wing Hang Cheung, Dino Samartzis, Keith Dip-Kei Luk \\ Department of Orthopaedics and Traumatology, The University of Hong Kong, Pokfulam, Hong Kong, SAR, China
}

\begin{abstract}
Study Design: Prospective study.
Purpose: To determine the risk of clinically significant curve progression in adolescent idiopathic scoliosis (AIS) based on the initial Cobb angle and to test the utility of the distal radius and ulna (DRU) classification in predicting these outcomes.

Overview of Literature: Determining the remaining growth potential in AIS patients is necessary for predicting prognosis and initiating treatment. Limiting the maturity Cobb angle to $<40^{\circ}$ and $<50^{\circ}$ reduces the risk of adulthood progression and need for surgery, respectively. The risk of curve progression is the greatest with skeletally immature patients and thus warrants close monitoring or early intervention. Many parameters exist for measuring the skeletal maturity status in AIS patients, but the DRU classification has been shown to be superior in predicting peak growth and growth cessation. However, its predictive capabilities for curve progression are unknown.

Methods: Totally, 513 AIS patients who presented with Risser 0-3 were followed until either skeletal maturity or the need for surgery, with a minimum 2-year follow-up period. Outcomes of $40^{\circ}$ and $50^{\circ}$ were used for probability analysis based on the cut-offs of adulthood progression risk and surgical threshold, respectively.

Results: At the R6/U5 grade, most curves (probability of $\geq 48.1 \%-55.5 \%$ ) beyond a Cobb angle of $25^{\circ}$ progressed to the $40^{\circ}$ threshold. For curves of $\geq 35^{\circ}$, there was a high risk of unfavorable outcomes, regardless of skeletal maturity. Most patients with the R9 grade did not progress, regardless of the initial curve magnitude (probability of $0 \%$ to reach the $50^{\circ}$ threshold for an initial Cobb angle of $\geq 35^{\circ}$ ).

Conclusions: This large-scale study illustrates the utility of the DRU classification for predicting curve progression and how it may effectively guide the timing of surgery. Bracing may be indicated for skeletally immature patients at an initial Cobb angle of $25^{\circ}$, and those with a scoliosis $\geq 35^{\circ}$ are at an increased risk of an unfavorable outcome, despite being near skeletal maturity.
\end{abstract}

Keywords: Adolescent idiopathic scoliosis; Curve progression; Growth; Distal radius and ulna classification

This is the 2017 APSS-Asian Spine Journal Best Paper Award.

\section{Received Jan 17, 2018; Accepted Feb 3, 2018}

Corresponding author: Jason Pui Yin Cheung

Department of Orthopaedics and Traumatology, The University of Hong Kong, Professorial Block, 5th Floor, 102 Pokfulam Road, Pokfulam, Hong Kong, SAR, China

Tel: +852-2255-4581, Fax: +852-2817-4392, E-mail: cheungjp@hku.hk 


\section{Introduction}

Determining a child's remaining skeletal growth is crucial for the management of adolescent idiopathic scoliosis (AIS) [1-3]. This is particularly important for brace treatment because the prompt initiation of this management approach can reduce the number of AIS patients undergoing surgery [4-6]. Generally, the goal of a bracing treatment is to prevent a patient from reaching the commonly described surgical threshold of $>50^{\circ}$. However, the prevention of virtually any Cobb angle progression by bracing is also beneficial. According to natural history studies, a smaller Cobb angle at skeletal maturity is more cosmetically acceptable and preventing the $40^{\circ}$ threshold can reduce the risk of adulthood deterioration and need for surgery [3,7]. Adulthood progression as reported in these studies can be at least $1^{\circ}$ per year for curvatures of $\geq 40^{\circ}$ at skeletal maturity.

Good brace compliance with more than 20 hours of daily use has been shown to be effective in reducing curve progression [8]. In a multicenter study, Weinstein et al. [6] demonstrated that in patients with high-risk curves, bracing significantly decreased curve progression based on a dose-effect response and, in turn, the need for surgery. Identification of peak height velocity (PHV) is thus essential for good outcomes as it indicates the period where the risk of deformity progression is the greatest. It is equally important to identify patients who have passed their growth spurt as brace effectiveness is limited in these cases and complications may arise from prolonged use [8-10]. Extensive and indiscriminate use of bracing in children has been shown to reduce spinal mobility, lead to poor body image and loss of self-esteem, and reduce the quality of life [11-13]. Maintaining good health and quality of life parameters is crucial to the success of AIS treatment [14-16].

It is the clinician's responsibility to gauge the risk of reaching the previously discussed thresholds and the potential benefits of prescribing brace treatment. Having an accurate skeletal maturity assessment tool is thus necessary to predict a patient's remaining growth potential to facilitate this decision-making process. To this end, prediction models can be very useful as they generate an algorithm based on large datasets [17]. However, a standardized parameter must be used for accurate testing. The distal radius and ulna (DRU) classification incorporates the whole range of growth phases with 11 radius grades (R1-R11) and 9 ulna grades (U1-U9). This classification

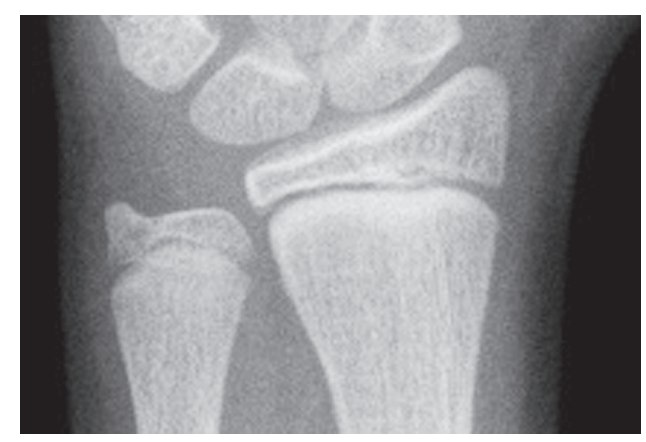

Fig. 1. Wrist radiograph showing a distal radius and ulna classification grade of R6 and U5, which indicates peak height velocity.

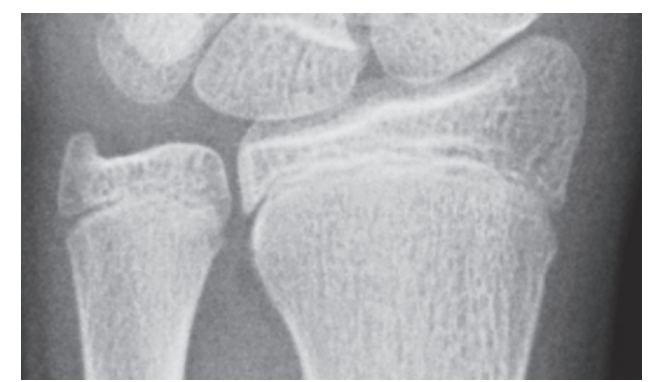

Fig. 2. Wrist radiograph showing a distal radius and ulna classification grade of R9 and U7, which indicates the end of significant growth.

has been shown to be easily reproducible, with excellent intra- and inter-rater reliability, and a simplified version has been developed for clinical use [18,19]. Due to being solely based on the morphology of two physeal plates, it is more user-friendly than measuring the entire hand. Large-scale thorough analyses of the relationship between the DRU classification and the Risser sign, age at menarche, and metacarpal/phalangeal physeal capping and fusion have been performed $[1,2,20]$, and it has been shown that the DRU classification is superior to conventional methods in determining peak growth (Fig. 1) and growth cessation (Fig. 2). Nevertheless, its utility in predicting curve progression and curve magnitude at skeletal maturity has not yet been determined. Therefore, this study aimed to investigate the capacity of the DRU classification, using the presenting Cobb angle, to predict whether the clinically relevant $40^{\circ}$ and $50^{\circ}$ outcome thresholds would be reached at skeletal maturity.

\section{Materials and Methods}

\section{Clinical data}

This study was based on prospectively collected data from 
a tertiary scoliosis specialty clinic. From April 1998 to June 2014, all AIS patients who initially presented with Risser $0-3$ and were less than 2 years post menarche were followed until skeletal maturity at Risser 5 or until surgical intervention was needed, which was offered for those with Cobb angles of $>50^{\circ}$. All non-idiopathic scoliosis patients and AIS patients who first presented with a Cobb angle of $\geq 40^{\circ}$ were excluded. This study was approved by a local ethics review board.

At initial presentation, age, body height (BH), arm span (AS), coronal Cobb angle magnitude, Lenke classification, Risser sign, and DRU classification grades were recorded. Age at menarche was listed for females. All patient data were recorded as per the standard protocol in our clinic. $\mathrm{BH}$ was measured with a patient standing on a stadiometer without shoes and socks. The heels were kept together, and the recorder measured only $\mathrm{BH}$ after the patient was asked to stand upright with the chin up. AS was measured with the patient's arms apart in the maximally outstretched position from the ends of the middle fingers. All Cobb angles were measured for all curves on a standing posteroanterior whole spine plain radiograph. The same radiograph was used for grading the Risser sign. The DRU classification was measured using left wrist radiographs routinely obtained for skeletal maturity grading [19]. Radiographs were generally obtained every 6 months, particularly during the peak growth spurt periods. It was also recorded whether bracing was prescribed for patients. Our protocol for bracing follows that of the SRS criteria, which includes patients aged 10 or older, with Risser 0-2, with a primary curve angle of $25^{\circ}-40^{\circ}$, and who were premenarchal or $<1$ year post menarche (females). However, due to the lack of objective compliance monitoring data and possible inaccuracies with self-reported compliance, this parameter was not used in the study.

The risk of curve progression was tested based on each patient's initial curve magnitude and DRU classification grade. The initial curve magnitudes were grouped as $\geq 10^{\circ}$ to $<15^{\circ}, \geq 15^{\circ}$ to $<20^{\circ}, \geq 20^{\circ}$ to $<25^{\circ}, \geq 25^{\circ}$ to $<30^{\circ}, \geq 30^{\circ}$ to $<35^{\circ}$, and $\geq 35^{\circ}$. The major curve Cobb angle was used for progression analysis in this study. The final Cobb angle at either skeletal maturity or at surgical intervention was used as the outcome measure. Curve progression was identified as any curve progression beyond $5^{\circ}$ of the initial curve magnitude. Curve progression to two "poor" outcomes was used for analysis. This included the $40^{\circ}$ threshold for increased risk of adulthood deterioration and the $50^{\circ}$ threshold for the need for surgery.

\section{Statistical analysis}

Descriptive data are presented as mean \pm standard deviation, and statistical analyses were performed using oneway analysis of variance to explore intergroup differences. For bracing patents, the chi-square test was utilized to determine the relationship between various parameters (i.e., bracing) and curve progression. Following univariate analyses, covariates were chosen for regression modelling if they had a $p$-value of $<0.200$. Multivariate stepwise logistic regression was used to determine the probabilities of curve progression to the outcomes of $40^{\circ}$ and $50^{\circ}$ thresholds. Two sets of analyses were undertaken by dichotomizing the presence or absence of progression to one of the thresholds based on the initial Cobb angle and DRU classification grade. The predicted percentage change generated from the regression model was used to represent the risk of progression. The forecasted probability was divided into quartiles: $<25 \%, 25 \%$ to $<50 \%, 50 \%$ to $<75 \%$, and $\geq 75 \%$; a probability of $25 \%$ to $<50 \%$ was considered moderate, while that of $\geq 50 \%$ was considered high risk. All $p$-values of $<0.05$ were regarded as statistically significant, and $95 \%$ confidence intervals were reported.

\section{Results}

Totally, 513 AIS patients ( $\mathrm{n}=400$ females) were studied. The mean age at initial presentation was $12.5 \pm 1.3$ years, and the mean follow-up duration was $4.7 \pm 2.5$ years until skeletal maturity or when surgery was needed. At initial presentation, $50.2 \%$ of the patients were premenarchal. Those who were postmenarchal presented an average of $8.1 \pm 6.1$ months after menarche. Baseline data are presented in Table 1. The spread of data was similar between females and males, and the two groups were combined for the general representation of the study population. Up to 316 patients (61.6\%) received bracing, and 32 of them eventually required surgery, despite bracing. A total of 226 patients (44.1\%) were at Risser 0, while 175 (34.1\%) were graded as R6 and 156 (30.4\%) as U5 at initial presentation. According to published data [1,2], an R6U5 grade indicates the peak growth period. Regarding curve types, patients with major thoracolumbar or lumbar curves had lesser risk of progression than those with major thoracic and double thoracic curves. Those with Lenke 5 curves 
Table 1. Baseline demographic data

\begin{tabular}{|c|c|c|c|}
\hline Characteristic & Total & Females & Males \\
\hline \multicolumn{4}{|l|}{ Risser stage } \\
\hline 0 & $226(44.1)$ & $180(45.0)$ & $46(40.7)$ \\
\hline 1 & $82(16.0)$ & $70(17.4)$ & $12(10.6)$ \\
\hline 2 & $100(19.5)$ & $81(20.3)$ & $19(16.8)$ \\
\hline 3 & $105(20.5)$ & $69(17.3)$ & $36(31.9)$ \\
\hline \multicolumn{4}{|l|}{ Modified Lenke curve type } \\
\hline 1 & $99(19.3)$ & $81(20.3)$ & $18(15.9)$ \\
\hline 2 & $35(6.8)$ & $22(5.5)$ & $13(11.5)$ \\
\hline 3 & $106(20.7)$ & $90(22.5)$ & $16(14.2)$ \\
\hline 4 & $10(1.9)$ & $5(1.3)$ & $5(4.4)$ \\
\hline 5 & $125(24.4)$ & $91(22.8)$ & $34(30.1)$ \\
\hline 6 & $138(26.9)$ & $111(27.8)$ & $27(23.9)$ \\
\hline Curve type changes upon maturity & $90(17.5)$ & - & - \\
\hline \multicolumn{4}{|l|}{ Final curve type } \\
\hline 1 & $82(16.0)$ & - & - \\
\hline 2 & $43(8.4)$ & - & - \\
\hline 3 & $126(24.6)$ & - & - \\
\hline 4 & $18(3.5)$ & - & - \\
\hline 5 & $110(21.4)$ & - & - \\
\hline 6 & $134(26.1)$ & - & - \\
\hline \multicolumn{4}{|l|}{ Radius grades } \\
\hline 5 & $19(3.7)$ & $11(2.7)$ & $8(7.1)$ \\
\hline 6 & $175(34.1)$ & 133 (33.3) & 42 (37.2) \\
\hline 7 & $92(17.9)$ & 74 (18.5) & 18 (15.9) \\
\hline 8 & $184(35.9)$ & $152(38.0)$ & 32 (28.3) \\
\hline 9 & $40(7.8)$ & $28(7.0)$ & $12(10.6)$ \\
\hline 10 & $3(0.6)$ & $2(0.5)$ & $1(0.9)$ \\
\hline \multicolumn{4}{|l|}{ Ulna grades } \\
\hline 4 & $40(7.8)$ & $30(7.5)$ & $10(8.8)$ \\
\hline 5 & $156(30.4)$ & $124(31.0)$ & 32 (28.3) \\
\hline 6 & $138(26.9)$ & $103(25.7)$ & $35(31.0)$ \\
\hline 7 & $158(30.8)$ & $130(32.5)$ & 28 (24.8) \\
\hline 8 & $21(4.1)$ & $13(3.3)$ & $8(7.1)$ \\
\hline \multicolumn{4}{|l|}{ Outcome at skeletal maturity } \\
\hline \multicolumn{4}{|l|}{ Curve progression $\left({ }^{\circ}\right)$} \\
\hline$<40$ & $152(29.6)$ & - & - \\
\hline$\geq 40$ & $268(52.2)$ & - & - \\
\hline$\geq 50$ & $74(14.4)$ & - & - \\
\hline No curve progression & $203(39.6)$ & - & - \\
\hline \multicolumn{4}{|l|}{ Initial curve magnitude $\left({ }^{\circ}\right)$} \\
\hline$\geq 10<15$ & $21(4.1)$ & - & - \\
\hline
\end{tabular}


Table 1. Continued

\begin{tabular}{cccc}
\hline Characteristic & Total & Females & Males \\
\hline $15<20$ & $72(14.0)$ & - & - \\
$\geq 20<25$ & $148(28.8)$ & - & - \\
$\geq 25<30$ & $141(27.5)$ & - & - \\
$\geq 30<35$ & $93(18.1)$ & - & - \\
$\geq 35$ & $38(7.4)$ & - & - \\
\hline
\end{tabular}

Values are presented as number (\%).

Table 2. Outcomes of bracing

\begin{tabular}{|c|c|c|c|c|c|c|c|}
\hline \multirow{2}{*}{ Initial Cobb angle $\left({ }^{\circ}\right)$} & \multirow{2}{*}{ Bracing } & \multicolumn{6}{|c|}{ Curve progression } \\
\hline & & No progression to $40^{\circ}$ & Yes $\geq 40^{\circ}$ & $p$-value & No progression to $50^{\circ}$ & Yes $\geq 50^{\circ}$ & $p$-value \\
\hline \multirow[t]{2}{*}{$\geq 10<15$} & Yes & $7(75.0)$ & $1(12.5)$ & 0.687 & $7(87.5)$ & $1(12.5)$ & 0.460 \\
\hline & No & $4(75.0)$ & 0 & & $4(100.0)$ & 0 & \\
\hline \multirow[t]{2}{*}{$\geq 15<20$} & Yes & $14(70.0)$ & $6(30.0)$ & $0.008^{a)}$ & $18(90.0)$ & $2(10.0)$ & 0.326 \\
\hline & No & $8(88.9)$ & $1(11.1)$ & & $9(100.0)$ & 0 & \\
\hline \multirow[t]{2}{*}{$\geq 20<25$} & Yes & $25(54.3)$ & $21(45.7)$ & $0.018^{\mathrm{a})}$ & $31(67.4)$ & $15(32.6)$ & $0.005^{a)}$ \\
\hline & No & $17(89.5)$ & $2(10.5)$ & & $19(100)$ & 0 & \\
\hline \multirow[t]{2}{*}{$\geq 25<30$} & Yes & $26(44.1)$ & $33(55.9)$ & $0.011^{\mathrm{a})}$ & 41 (69.5) & $18(30.5)$ & 0.087 \\
\hline & No & $7(100.0)$ & 0 & & $7(100.0)$ & 0 & \\
\hline \multirow[t]{2}{*}{$\geq 30<35$} & Yes & $13(39.4)$ & $20(60.6)$ & 0.205 & $25(75.8)$ & $8(24.2)$ & 0.334 \\
\hline & No & $2(66.7)$ & $1(33.3)$ & & $3(100.0)$ & 0 & \\
\hline \multirow[t]{2}{*}{$\geq 35$} & Yes & $7(43.7)$ & $9(56.3)$ & - & $11(68.7)$ & $5(31.3)$ & - \\
\hline & No & 0 & 0 & & 0 & 0 & \\
\hline
\end{tabular}

Values are presented as number (row \%).

a) Statistical significance.

rarely $(6.4 \%)$ progressed to the surgical threshold.

Specifically regarding the 226 patients with Risser 0 at baseline, $182(80.5 \%)$ received bracing treatment, and of these, $20(11.0 \%)$ subsequently underwent surgery. The outcomes from bracing are listed in Table 2. Up to $56.3 \%$ of the patients with an initial Cobb angle of $\geq 35^{\circ}$ reached both $40^{\circ}$ and $50^{\circ}$ thresholds, despite most of them being braced. Differences in the spread of parameters were observed between those who did not have curve progression at all or did not reach clinically significant outcome thresholds and those who reached the $40^{\circ}$ or $50^{\circ}$ threshold; these data are presented in Table 3. Of all patients at Risser 0, only 58 (25.7\%) did not experience curve progression; of them, 38 (65.5\%) were braced. A classification of Risser 0 was a significant predictor of later reaching the surgical threshold, but a classification of up to Risser 3 can still have significant risk for adulthood progression. A statistically significant prediction model could not be constructed for the Risser sign. Although 168 patients (74.3\%) had significant curve progression, 72 (42.9\%) did not progress beyond the $40^{\circ}$ threshold, regardless of the initial Cobb angle. A total of 96 patients (57.1\%) reached the $40^{\circ}$ threshold and $51(30.4 \%)$ reached the $50^{\circ}$ threshold.

In general, those who presented with larger Cobb angles were more likely to reach the $40^{\circ}$ or $50^{\circ}$ threshold (Fig. $3)$. At the final outcome point, 162 (31.6\%) reached the $40^{\circ}$ threshold and $74(14.4 \%)$ reached the $50^{\circ}$ threshold. The Cobb angle for 148 of our patient $(28.8 \%)$ was $\geq 20^{\circ}$ to $<25^{\circ}$ at initial presentation. The regression model for curve progression to the $40^{\circ}$ threshold is presented in Tables 4, 5. The overall correct predictions for the radius and ulna classification grades were $74.5 \%$ and $71.5 \%$, respectively. Similarly, the regression model (Tables 6, 7) for curve progression to the $50^{\circ}$ threshold had high overall 
Table 3. Factors related to curve progression

\begin{tabular}{|c|c|c|c|c|c|c|}
\hline Variable & $\begin{array}{c}\text { No curve } \\
\text { progression to } 40^{\circ}\end{array}$ & $\begin{array}{c}\text { Curve } \\
\text { progression } \geq 40^{\circ}\end{array}$ & $p$-value & $\begin{array}{c}\text { No curve } \\
\text { progression to } 50^{\circ}\end{array}$ & $\begin{array}{c}\text { Curve } \\
\text { progression } \geq 50^{\circ}\end{array}$ & $p$-value \\
\hline Menarche & & & $<0.001^{\text {a) }}$ & & & $<0.001^{\mathrm{a})}$ \\
\hline Yes & $149(74.9)$ & $50(25.1)$ & & $183(92.0)$ & $16(8.0)$ & \\
\hline No & $124(61.7)$ & 77 (38.3) & & $158(78.6)$ & $43(21.4)$ & \\
\hline Risser stage & & & $<0.001^{\text {a) }}$ & & & $<0.001^{\text {a) }}$ \\
\hline 0 & $130(57.5)$ & $96(42.5)$ & & $175(77.4)$ & $51(22.6)$ & \\
\hline 1 & $60(70.7)$ & $22(26.8)$ & & $75(91.5)$ & $7(8.5)$ & \\
\hline 2 & $79(79.0)$ & $21(21.0)$ & & $92(92.0)$ & $8(8.0)$ & \\
\hline 3 & $85(80.0)$ & $20(19.0)$ & & $97(92.4)$ & $8(7.6)$ & \\
\hline Modified Lenke curve type & & & $0.006^{\mathrm{al}}$ & & & 0.012 \\
\hline 1 & $61(61.6)$ & $38(38.4)$ & & 79 (79.8) & $20(20.2)$ & \\
\hline 2 & $25(71.4)$ & $10(28.6)$ & & $30(85.7)$ & $5(14.3)$ & \\
\hline 3 & $67(34.0)$ & 39 (36.8) & & $90(84.9)$ & $16(15.1)$ & \\
\hline 4 & $5(50.0)$ & $5(50.0)$ & & $6(60.0)$ & $4(40.0)$ & \\
\hline 5 & 104 (83.2) & $21(16.8)$ & & $117(93.6)$ & $8(6.4)$ & \\
\hline 6 & $92(66.7)$ & 46 (33.3) & & 117 (84.8) & $21(15.2)$ & \\
\hline Radius grades & & & $<0.001^{\text {a) }}$ & & & $0.002^{a)}$ \\
\hline 5 & 12 (63.2) & $7(36.8)$ & & 14 (73.6) & $5(26.3)$ & \\
\hline 6 & 99 (56.6) & $76(43.4)$ & & $137(78.3)$ & $38(21.7)$ & \\
\hline 7 & $67(72.8)$ & $25(27.2)$ & & $83(90.2)$ & $9(9.8)$ & \\
\hline 8 & $137(74.5)$ & $47(25.5)$ & & $162(88.0)$ & $22(12.0)$ & \\
\hline 9 & $36(90.0)$ & $4(10.0)$ & & $40(100.0)$ & $0(0.0)$ & \\
\hline 10 & $3(100.0)$ & $0(0.0)$ & & $3(100.0)$ & $0(0.0)$ & \\
\hline Ulna grades & & & $<0.001^{\text {a) }}$ & & & $<0.001^{\text {a) }}$ \\
\hline 4 & $21(52.5)$ & $19(47.5)$ & & $29(72.5)$ & $11(27.5)$ & \\
\hline 5 & $94(60.3)$ & 62 (39.7) & & 124 (79.5) & $32(20.5)$ & \\
\hline 6 & 96 (69.6) & $42(30.4)$ & & 123 (89.1) & $15(10.9)$ & \\
\hline 7 & $128(81.0)$ & $30(19.0)$ & & 145 (91.8) & $13(8.2)$ & \\
\hline 8 & $15(71.4)$ & $6(28.6)$ & & $18(85.7)$ & $3(14.3)$ & \\
\hline Initial curve magnitude $\left(^{\circ}\right)$ & & & $<0.001^{\text {a) }}$ & & & $<0.001^{\text {a) }}$ \\
\hline$\geq 10<15$ & $19(90.5)$ & $2(9.5)$ & & $20(95.2)$ & $1(4.8)$ & \\
\hline$\geq 15<20$ & $63(87.5)$ & $9(12.5)$ & & $68(94.4)$ & $4(5.6)$ & \\
\hline$\geq 20<25$ & $120(81.1)$ & $28(18.9)$ & & 133 (89.9) & $15(10.1)$ & \\
\hline$\geq 25<30$ & $87(61.7)$ & $54(38.3)$ & & $117(83.0)$ & $24(17.0)$ & \\
\hline$\geq 30<35$ & $50(53.8)$ & $43(46.2)$ & & 77 (82.8) & $16(17.2)$ & \\
\hline$\geq 35$ & 15 (39.5) & $23(60.5)$ & & $24(63.2)$ & 14 (36.8) & \\
\hline
\end{tabular}

Values are presented as number (\%).

a) Statistical significance.

prediction accuracies of $85.6 \%$ and $86.0 \%$ for the radius and ulna classification grades, respectively. At R6 and U5, most curves with a Cobb angle of $>25^{\circ}$ progressed, while at R8 and U7, only those with a Cobb angle of $\geq 35^{\circ}$ were at risk of unfavorable outcomes. At R9 and U8, most curves did not progress. A simple summary of the 


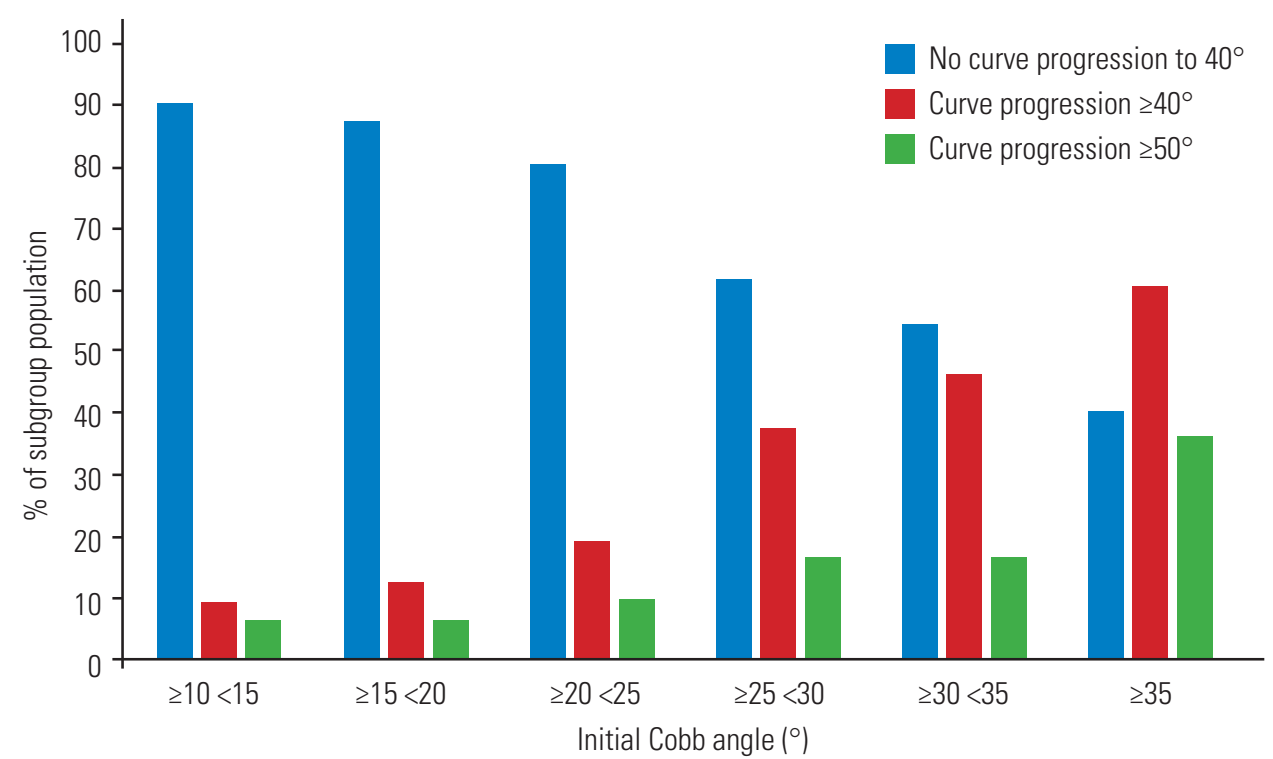

Fig. 3. Curve progression (in percentages) based on the Cobb angle at initial presentation.

Table 4. 40-degree threshold regression model (radius)

\begin{tabular}{|c|c|c|c|c|c|c|}
\hline \multirow{2}{*}{$\begin{array}{l}\text { Initial curve } \\
\text { magnitude }\left({ }^{\circ}\right)\end{array}$} & \multicolumn{6}{|c|}{ Radius } \\
\hline & $\mathrm{R5}$ & $\mathrm{R6}$ & $\mathrm{R7}$ & R8 & $\mathrm{Rg}$ & R10 \\
\hline$\geq 10<15$ & $0 / 3,0,12.8$ & $1 / 8,-10$ to $35,14.6$ & $0 / 2,0,6.4$ & $0 / 4,0,5.9$ & $1 / 4,42$ to $67,2.1$ & - \\
\hline$\geq 15<20$ & $1 / 5,-15$ to $55,17.6$ & $4 / 26,2$ to $29,20.0$ & $2 / 13,-4$ to $35,9.1$ & $2 / 18,-3$ to $26,8.3$ & $0 / 8,0,3.0$ & $0 / 2,0,0$ \\
\hline$\geq 20<25$ & $2 / 2,100.26 .2$ & $16 / 53,18$ to $43,29.4$ & $7 / 25,10$ to $46,14.3$ & $3 / 61,-1$ to $10,13.1$ & $0 / 7,0,4.8$ & - \\
\hline$\geq 25<30$ & $2 / 5,-3$ to $83,51.6$ & $27 / 44,47$ to $76,55.5$ & $8 / 28,12$ to $45,33.4$ & $17 / 52,20$ to $45,31.2$ & $0 / 11,0,13.3$ & $0 / 1,0,0$ \\
\hline$\geq 30<35$ & $1 / 2,-19$ to $119,60.5$ & $20 / 30,50$ to $84,64.2$ & $5 / 18,7$ to $48,41.8$ & $14 / 34,25$ to $58,39.4$ & $3 / 9,3$ to $64,18.0$ & - \\
\hline$\geq 35$ & $1 / 2,-19$ to $119,71.2$ & $8 / 14,31$ to $83,74.3$ & $3 / 6,10$ to $90,53.8$ & $11 / 15,51$ to $96,51.2$ & $0 / 1,0,26.2$ & - \\
\hline
\end{tabular}

Values are presented as number of patients with curve progression $\geq 40 \%$ (total number of patients), $95 \%$ confidence interval for population proportion, and predicted \% generated from regression model. Bold typed cells represent the radius grades and curve magnitude at initial presentation for which curve progression to $\geq 40^{\circ}$ are likely to happen at a probability of $\geq 25 \%$. those with wide $95 \%$ confidence intervals can be resulted from the few patients for accurate estimates.

Table 5. 40-degree threshold regression model (ulna)

\begin{tabular}{|c|c|c|c|c|c|}
\hline \multirow{2}{*}{$\begin{array}{l}\text { Initial curve } \\
\text { magnitude }\left({ }^{\circ}\right)\end{array}$} & \multicolumn{5}{|c|}{ Ulna } \\
\hline & U4 & U5 & U6 & U7 & U8 \\
\hline$\geq 10<15$ & $0 / 5,0,19.3$ & $1 / 6,-13$ to $47,10.2^{a)}$ & $0 / 2,0,6.6$ & $1 / 7,-12$ to $40,3.4^{\text {a) }}$ & $0 / 1,0,5.6$ \\
\hline$\geq 15<20$ & $2 / 8,-5$ to $55,27.4^{\text {a) }}$ & $3 / 24,-1$ to $26,15.2^{\text {a) }}$ & $4 / 20,2$ to $38,10.0$ & $0 / 16,0,5.2$ & $0 / 4,0,8.6$ \\
\hline$\geq 20<25$ & $7 / 12,30$ to $86,42.3$ & $10 / 40,12$ to $38,25.8$ & $8 / 39,8$ to $33,17.7$ & $3 / 53,-1$ to $12,9.6^{\text {a) }}$ & $0 / 4,0,15.5$ \\
\hline$\geq 25<30$ & $6 / 10,30$ to $90,66.2$ & $24 / 46,38$ to $67,48.1$ & $15 / 41,22$ to $51,36.5$ & $7 / 39,6$ to $30,22.1$ & $2 / 5,-3$ to $83,32.8^{\text {a) }}$ \\
\hline$\geq 30<35$ & $1 / 1,100,75.7$ & $17 / 28,43$ to $79,59.6$ & $13 / 30,26$ to $61,47.8$ & $10 / 29,17$ to $52,31.2$ & $2 / 5,-3$ to $43,43.8^{\text {a) }}$ \\
\hline$\geq 35$ & $3 / 4,33$ to $117,84.4$ & $7 / 12,30$ to $86,71.9$ & $2 / 6,-4$ to $71,61.4^{a)}$ & $9 / 14,39$ to $89,44.0$ & $2 / 2,100,57.5^{\mathrm{a})}$ \\
\hline
\end{tabular}

Values are presented as number of patients with curve progression $\geq 40 \%$ (total number of patients), $95 \%$ confidence interval for population proportion, and predicted \% generated from regression model. Bold typed cells represent the radius grades and curve magnitude at initial presentation for which curve progression to $\geq 40^{\circ}$ are likely to happen at a probability of $\geq 25 \%$.

${ }^{\text {al }}$ Those with wide $95 \%$ confidence intervals can be resulted from the few patients for accurate estimates. 
Table 6. 50-degree threshold regression model (radius)

\begin{tabular}{|c|c|c|c|c|c|c|}
\hline \multirow{2}{*}{$\begin{array}{l}\text { Initial curve } \\
\text { magnitude }\left({ }^{\circ}\right)\end{array}$} & \multicolumn{6}{|c|}{ Radius } \\
\hline & $\mathrm{R} 5$ & $\mathrm{R} 6$ & $\mathrm{R} 7$ & $\mathrm{R} 8$ & $\mathrm{R} 9$ & R10 \\
\hline$\geq 10<15$ & $0 / 3,0,9.7$ & $1 / 8,-10$ to $35,6.7$ & $0 / 2,0,2.5$ & $0 / 4,0,3.1$ & $0 / 4,0,0$ & - \\
\hline$\geq 15<20$ & $1 / 5,14$ to $26,12.3$ & $1 / 26,-4$ to $11,8.6$ & $1 / 13,-7$ to $22,3.3$ & $1 / 18,-5$ to $16,4.0$ & $0 / 8,0,0$ & $0 / 2,0,0$ \\
\hline$\geq 20<25$ & $0 / 2,0,21.8$ & $11 / 53,10$ to $32,15.8$ & $3 / 25,-1$ to $25,6.3$ & $1 / 61,0$ to $12,7.6$ & $0 / 7,0,0$ & - \\
\hline$\geq 25<30$ & $2 / 5,-3$ to $83,35.2^{\text {a) }}$ & $14 / 44,18$ to $46,26.8$ & $3 / 28,-1$ to $22,11.6^{\text {a) }}$ & $5 / 52,2$ to $18,13.9$ & $0 / 11,0,0$ & $0 / 1,0,0$ \\
\hline$\geq 30<35$ & $1 / 2,-19$ to $119,36.1^{\text {a) }}$ & $7 / 30,8$ to $38,27.5$ & $1 / 18,-5$ to $16,12.0^{\text {a) }}$ & $7 / 34,2$ to $39,14.3$ & $0 / 9,0,0$ & - \\
\hline$\geq 35$ & $1 / 2,-19$ to $119,58.7^{\text {a) }}$ & $4 / 14,5$ to $52,48.9$ & $1 / 6,-13$ to $47,25.6^{\text {a) }}$ & $8 / 15,28$ to $79,29.6$ & $0 / 1,0,0$ & - \\
\hline
\end{tabular}

Values are presented as number of patients with curve progression $\geq 50^{\circ}$ (total number of patients), $95 \%$ confidence interval for population proportion, and predicted \% generated from regression model. Bold typed cells represent the radius grades and curve magnitude at initial presentation for which curve progression to $\geq 50^{\circ}$ are likely to happen at a probability of $\geq 25 \%$.

alThose with wide $95 \%$ confidence intervals can be resulted from the few patients for accurate estimates.

Table 7. 50-degree threshold regression model (ulna)

\begin{tabular}{|c|c|c|c|c|c|}
\hline \multirow{2}{*}{$\begin{array}{l}\text { Initial curve } \\
\text { magnitude }\left({ }^{\circ}\right)\end{array}$} & \multicolumn{5}{|c|}{ Ulna } \\
\hline & U4 & U5 & U6 & U7 & U8 \\
\hline$\geq 10<15$ & $0 / 5,0,9.5$ & $1 / 6,-13$ to $46,5.3^{\text {a) }}$ & $0 / 2,0,2.6$ & $0 / 7,0,1.8$ & $0 / 1,0,3.3$ \\
\hline$\geq 15<20$ & $1 / 8,-10$ to $35,12.6^{\text {a) }}$ & $2 / 24,-3$ to $19,7.2^{\text {a) }}$ & $1 / 20,28$ to $72,3.5$ & $0 / 16,0,2.4$ & $0 / 4,0,4.5$ \\
\hline$\geq 20<25$ & $4 / 12,7$ to $60,24.5$ & $7 / 40,6$ to $29,14.8$ & $4 / 39,1$ to $20,7.5$ & $0 / 53,0,5.3$ & $0 / 4,0,9.6$ \\
\hline$\geq 25<30$ & $3 / 10,2$ to $58,36.5$ & $13 / 46,-13$ to $70,23.6^{\text {a) }}$ & $5 / 41,2$ to $22,12.6$ & $2 / 39,-2$ to $12,9.0^{a)}$ & $1 / 5,14$ to $26,15.8$ \\
\hline$\geq 30<35$ & $0 / 1,0,40.1$ & $6 / 28,6$ to $37,26.4$ & $5 / 30,3$ to $30,14.4$ & $5 / 29,4$ to $31,10.3$ & $0 / 5,0,17.9$ \\
\hline$\geq 35$ & $3 / 4,33$ to $117,63.2$ & $3 / 12,1$ to $50,48.0$ & $0 / 6,0,30.1^{\text {a) }}$ & $6 / 14,17$ to $69,22.8$ & $2 / 2,100,35.9$ \\
\hline
\end{tabular}

Values are presented as number of patients with curve progression $\geq 50^{\circ} /$ (total number of patients), $95 \%$ confidence interval for population proportion, and predicted \% generated from regression model. Bold typed cells represent the radius grades and curve magnitude at initial presentation for which curve progression to $\geq 50^{\circ}$ are likely to happen at a probability of $\geq 25 \%$.

alThose with wide 95\% confidence intervals can be resulted from the few patients for accurate estimates.

Table 8. Summary of progression risk

\begin{tabular}{|c|c|c|c|c|}
\hline Skeletal maturity & Distal radius and ulna grades & Initial Cobb angle $\left({ }^{\circ}\right)$ & Progression to $40^{\circ}(\%)$ & Progression to $50^{\circ}(\%)$ \\
\hline \multirow[t]{4}{*}{ Immature } & R6 & $\geq 20<25$ & 29.4 & 15.8 \\
\hline & U5 & & 25.8 & 14.8 \\
\hline & R6 & $\geq 35$ & 74.3 & 48.9 \\
\hline & U5 & & 71.9 & 48.0 \\
\hline \multirow[t]{4}{*}{ Mature } & R9 & $\geq 20<25$ & 4.8 & 0 \\
\hline & U7 & & 9.6 & 5.3 \\
\hline & R9 & $\geq 35$ & 26.2 & 0 \\
\hline & U7 & & 44.0 & 22.8 \\
\hline
\end{tabular}

Immaturity and maturity defined as R6/U5 and R9/U7 as previously described. 


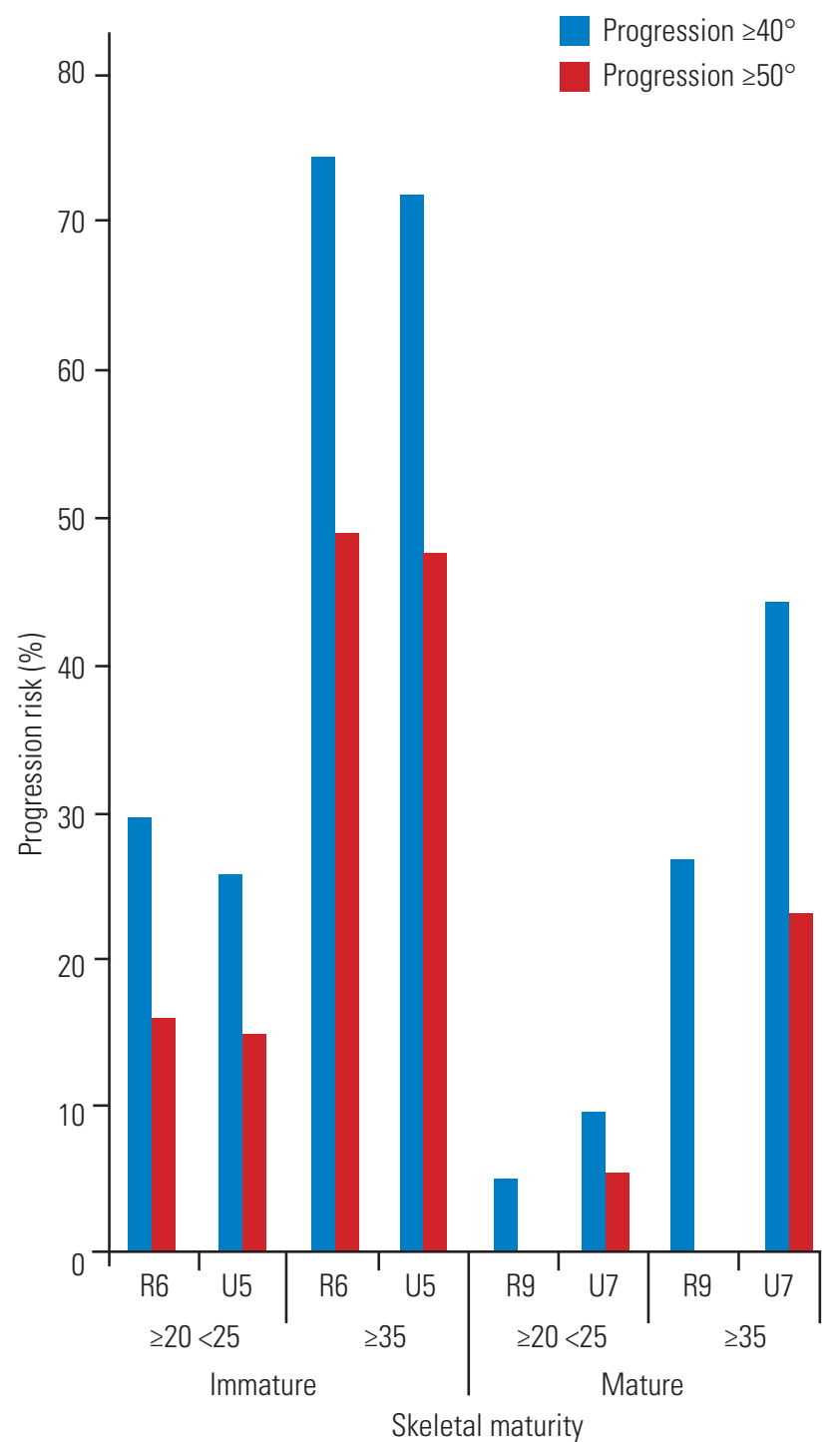

Fig. 4. Summary of curve progression risk for immature and mature patients based on the distal radius and ulna classification grade.

relationship between the initial Cobb angle and the DRU classification grade is illustrated in Table 8 and Fig. 4 .

\section{Discussion}

The present study illustrates the utility of the DRU classification grade to predict curve magnitude outcomes at skeletal maturity. Based on a large sample of AIS patients, the DRU classification grades together with the corresponding initial Cobb angles were effectively predictive of the outcomes at skeletal maturity. Using both radius and ulna grades for prediction may provide the best forecast for future curve progression. The present study also utilized clinically relevant curve progression outcomes for Cobb angles of $40^{\circ}$ or $50^{\circ}$. According to natural history studies, reaching either of these two values can be considered as poor outcomes due to the risks of adulthood deterioration and need for surgery $[3,7]$.

The accurate prediction of which AIS patients will experience curve progression is dependent on the clinician's ability to interpret the remaining growth potential. At initial presentation, the severity of the deformity measured by the Cobb angle and growth rate can provide useful information regarding the likely future curve behavior [5,21]. Many radiographic parameters exist to predict growth but have known limitations, including poor prediction of PHV for the timing of brace treatment [22-26], concerns with inter-observer reliability and complexity [27-29], and the cumbersome nature of using them in a busy clinical setting [30]. In contrast, the DRU classification has been shown to be reproducible and have excellent intra- and inter-rater reliability and the capability to be simplified for clinical use $[18,19]$. The DRU classification was found to be superior to the Risser sign, with its larger spread of grades prior to PHV so that both the acceleration and deceleration phases of growth can be determined during puberty [1]. The DRU classification has also been used to identify mismatches between growth and curve progression rates, which are not reported in other maturity parameters such as TW, Sanders stage, or Risser sign [20]. Hence, it is useful for determining the timing of both brace initiation and weaning. It has also been shown in this study that the DRU classification is superior to the Risser sign in terms of prediction, as patients with Risser 0 had near equal risk of adulthood progression and surgical threshold and those with Risser 3 still had a significant risk. This may be related to the unreliable Risser sign and mismatches observed with growth [1].

The risk of curve progression at PHV is of particular concern as prompt brace treatment at this point has been shown to reduce the number of AIS patients undergoing surgery [4-6]. From the analysis of those at PHV with R6/ U5 [1], patients with a large Cobb angle of $\geq 35^{\circ}$ at initial presentation are expected to reach both $40^{\circ}$ and $50^{\circ}$ thresholds with high probability (around 50\%-70\%). This suggests that the prognosis of patients who present with this curve magnitude at skeletal immaturity is poor. These patients should be provided brace treatment as soon as possible but are nevertheless likely to require surgical correction in the future. Even at R8/U7, which is near the expected growth cessation stage (R9/U7) [1], having such an 
initial curve magnitude will result in high (44.0\%-51.2\%) and moderate $(22.8 \%-29.6 \%)$ predicted probabilities of reaching the $40^{\circ}$ and $50^{\circ}$ thresholds, respectively. This suggests that bracing intervention is still indicated even at near skeletal maturity for large curves to prevent these poor outcomes. A more aggressive bracing protocol should be considered in these patients.

It is fortunate that only a small number of patients in the general population (7.4\%) present with a curve magnitude of $\geq 35^{\circ}$ because of our long-standing school screening program. Most patients in our study presented with an initial Cobb angle of $\geq 20^{\circ}$ to $<25^{\circ}(28.8 \%)$ or $\geq 25^{\circ}$ to $30^{\circ}(27.5 \%)$. Based on our results, the $25^{\circ}$ threshold is the main cut-off for the likelihood of poor outcomes in our scoliosis patients at PHV (R6/U5) by reaching the $40^{\circ}$ threshold. This is evidenced by only a $25.8 \%-29.4 \%$ probability in the $\geq 20^{\circ}$ to $<25^{\circ}$ group with an increase to $48.1 \%-55.5 \%$ probability in the $\geq 25^{\circ}$ to $<30^{\circ}$ group. However, patients are less likely to reach the surgical threshold, with only $14.8 \%-15.8 \%$ probability if they are in the $\geq 20^{\circ}$ to $<25^{\circ}$ group and a $23.6 \%-26.8 \%$ probability in the $\geq 25^{\circ}$ to $<30^{\circ}$ group. Hence, initial presenters at approximately $25^{\circ}$ and at PHV should be monitored closely for any deterioration. Bracing for this group should be considered only for those who experience curve progression during follow-up as the probability of reaching the surgical threshold is not high. No significant probability of poor outcomes was observed for either group in patients who present at a more mature stage of $\mathrm{R} 9$.

It is important to note that $61.6 \%$ of the patients in the present series were referred for bracing. As seen in Table 2 , many of those who were braced had poor outcomes. Unfortunately, due to lack of objective pressure or thermal sensors at the initiation of this study, we did not have compliance data to rigorously investigate the effects of bracing. Despite not having this information due to the study design, the probabilities presented are likely to be an underestimation of the true likelihood of reaching the $40^{\circ}$ and $50^{\circ}$ thresholds as these forecasts are based on the initial Cobb value rather than the treatment effect of bracing. Undoubtedly, with bracing, the probabilities will be more accurate and as such will require further studies. Nevertheless, one must be cautious to not underestimate the likelihood of poor outcomes. Despite not knowing the true bracing compliance for our patients, one is prudent to error on the side of relative overtreatment. However, this may be only true in the large initial Cobb angle group as the likelihood for reaching the surgical threshold is low for the $\leq 25^{\circ}$ groups at the time of PHV. In addition, the indiscriminate use of bracing may cause physical and mental harm with spinal stiffness, poorer body image, worse self-esteem, and quality of life [11-13].

There are several limitations to this study. Most significant is the lack of objective compliance data, which suggests that the probability data are an underestimation of the true risk of reaching poor outcomes. Nevertheless, the key objective of this study is to test the prediction power of the DRU classification based on the initial curve magnitude for determining outcomes. However, it appears that the radius has higher utility with a larger range of grades, including R9, which indicates a significant reduction in the risk of reaching a poor outcome. Another important limitation of the study is the assumption that curve progression patterns are similar among AIS patients, which is unlikely to be the case. Hence, the likelihood for successful bracing cannot rely only on the initial Cobb angle and DRU classification grade. Curve type may also influence the risk. Most patients with major thoracolumbar or lumbar curves do not progress to these clinically significant thresholds. It is likely that brace treatment is more effective in these curves than in those with thoracic curves [31]. The natural course of progression in AIS is not uniform, and some patients may not experience curve progression throughout growth, while others may only experience early or late deterioration. Hence, the results of this study do not indicate the duration of brace wear required. In addition, curve flexibility has been shown to greatly influence the outcomes of bracing and surgery [32-35]. This aspect of bracing, as well as the varied responses of different curve patterns, is beyond the intent of this study and should be addressed in future studies.

\section{Conclusions}

This research has shown the utility of using the DRU classification and initial Cobb angle to help forecast the likely outcome of AIS patients at skeletal maturity. With a better forecasting system for growth and curve progression, clinicians may be able differentiate which patients best respond to brace treatment without resorting to indiscriminate brace prescription and subjecting some patients to unnecessary bracing. Future studies should focus on specific effectiveness studies of bracing at certain maturity stages with proper compliance monitoring. 


\section{Conflict of Interest}

No potential conflict of interest relevant to this article was reported.

\section{References}

1. Cheung JP, Cheung PW, Samartzis D, Cheung KM, Luk KD. The use of the distal radius and ulna classification for the prediction of growth: peak growth spurt and growth cessation. Bone Joint J 2016;98B:1689-96.

2. Cheung JPY, Luk KD. Managing the pediatric spine: growth assessment. Asian Spine J 2017;11:804-16.

3. Weinstein SL, Dolan LA, Spratt KF, Peterson KK, Spoonamore MJ, Ponseti IV. Health and function of patients with untreated idiopathic scoliosis: a 50-year natural history study. JAMA 2003;289:559-67.

4. Sanders JO, Newton PO, Browne RH, Herring AJ. Bracing in adolescent idiopathic scoliosis, surrogate outcomes, and the number needed to treat. J Pediatr Orthop 2012;32 Suppl 2:S153-7.

5. Sitoula P, Verma K, Holmes L Jr, et al. Prediction of curve progression in idiopathic scoliosis: validation of the Sanders Skeletal Maturity Staging System. Spine (Phila Pa 1976) 2015;40:1006-13.

6. Weinstein SL, Dolan LA, Wright JG, Dobbs MB. Effects of bracing in adolescents with idiopathic scoliosis. N Engl J Med 2013;369:1512-21.

7. Weinstein SL, Ponseti IV. Curve progression in idiopathic scoliosis. J Bone Joint Surg Am 1983;65:44755.

8. Rahman T, Bowen JR, Takemitsu M, Scott C. The association between brace compliance and outcome for patients with idiopathic scoliosis. J Pediatr Orthop 2005;25:420-2.

9. Katz DE, Durrani AA. Factors that influence outcome in bracing large curves in patients with adolescent idiopathic scoliosis. Spine (Phila Pa 1976) 2001;26:2354-61.

10. Landauer F, Wimmer C, Behensky H. Estimating the final outcome of brace treatment for idiopathic thoracic scoliosis at 6-month follow-up. Pediatr Rehabil 2003;6:201-7.

11. Cheung KM, Cheng EY, Chan SC, Yeung KW, Luk $\mathrm{KD}$. Outcome assessment of bracing in adolescent idiopathic scoliosis by the use of the SRS-22 ques- tionnaire. Int Orthop 2007;31:507-11.

12. Ugwonali OF, Lomas G, Choe JC, et al. Effect of bracing on the quality of life of adolescents with idiopathic scoliosis. Spine J 2004;4:254-60.

13. Vasiliadis E, Grivas TB, Savvidou O, Triantafyllopoulos G. The influence of brace on quality of life of adolescents with idiopathic scoliosis. Stud Health Technol Inform 2006;123:352-6.

14. Wong CK, Cheung PW, Samartzis D, et al. Mapping the SRS-22r questionnaire onto the EQ-5D-5L utility score in patients with adolescent idiopathic scoliosis. PLoS One 2017;12:e175847.

15. Cheung PW, Wong CK, Lau ST, Cheung JP. Responsiveness of the EuroQoL 5-dimension (EQ5D) in adolescent idiopathic scoliosis. Eur Spine J 2018;27:278-85.

16. Cheung PW, Wong CK, Samartzis D, et al. Psychometric validation of the EuroQoL 5-dimension 5-level (EQ-5D-5L) in Chinese patients with adolescent idiopathic scoliosis. Scoliosis Spinal Disord 2016;11:19.

17. Deng L, Hu Y, Cheung JP, Luk KD. A data-driven decision support system for scoliosis prognosis. IEEE Access 2017;5:7874-84.

18. Cheung JP, Samartzis D, Cheung PW, Cheung KM, Luk KD. Reliability analysis of the distal radius and ulna classification for assessing skeletal maturity for patients with adolescent idiopathic scoliosis. Global Spine J 2016;6:164-8.

19. Cheung JP, Samartzis D, Cheung PW, Leung KH, Cheung KM, Luk KD. The distal radius and ulna classification in assessing skeletal maturity: a simplified scheme and reliability analysis. J Pediatr Orthop B 2015;24:546-51.

20. Cheung JP, Cheung PW, Samartzis D, Luk KD. Curve progression in adolescent idiopathic scoliosis does not match skeletal growth. Clin Orthop Relat Res 2018;476:429-36.

21. Karol LA, Virostek D, Felton K, Jo C, Butler L. The effect of the Risser stage on bracing outcome in adolescent idiopathic scoliosis. J Bone Joint Surg Am 2016;98:1253-9.

22. Biondi J, Weiner DS, Bethem D, Reed JF 3rd. Correlation of Risser sign and bone age determination in adolescent idiopathic scoliosis. J Pediatr Orthop 1985;5:697-701.

23. Dhar S, Dangerfield PH, Dorgan JC, Klenerman 
L. Correlation between bone age and Risser's sign in adolescent idiopathic scoliosis. Spine (Phila Pa 1976) 1993;18:14-9.

24. Hoppenfeld S, Lonner B, Murthy V, Gu Y. The rib epiphysis and other growth centers as indicators of the end of spinal growth. Spine (Phila Pa 1976) 2004;29:47-50.

25. Little DG, Sussman MD. The Risser sign: a critical analysis. J Pediatr Orthop 1994;14:569-75.

26. Shuren N, Kasser JR, Emans JB, Rand F. Reevaluation of the use of the Risser sign in idiopathic scoliosis. Spine (Phila Pa 1976) 1992;17:359-61.

27. Tanner JM, Whitehouse RH. Clinical longitudinal standards for height, weight, height velocity, weight velocity, and stages of puberty. Arch Dis Child 1976;51:170-9.

28. Tanner JM, Whitehouse RH, Hughes PC, Carter BS. Relative importance of growth hormone and sex steroids for the growth at puberty of trunk length, limb length, and muscle width in growth hormonedeficient children. J Pediatr 1976;89:1000-8.

29. Tanner JM, Whitehouse RH, Marubini E, Resele LF. The adolescent growth spurt of boys and girls of the Harpenden growth study. Ann Hum Biol 1976;3:10926.

30. Sanders JO, Khoury JG, Kishan S, et al. Predict- ing scoliosis progression from skeletal maturity: a simplified classification during adolescence. J Bone Joint Surg Am 2008;90:540-53.

31. Thompson RM, Hubbard EW, Jo CH, Virostek D, Karol LA. Brace success is related to curve type in patients with adolescent idiopathic scoliosis. J Bone Joint Surg Am 2017;99:923-8.

32. Cheung JP, Yiu KK, Vidyadhara S, Chan PP, Cheung PW, Mak KC. Predictability of supine radiographs for determining in-brace correction for adolescent idiopathic scoliosis. Spine (Phila $\mathrm{Pa}$ 1976) 2017 Dec 1 [Epub]. https://doi.org/10.1097/ BRS.0000000000002503.

33. Eyvazov K, Samartzis D, Cheung JP. The association of lumbar curve magnitude and spinal range of motion in adolescent idiopathic scoliosis: a cross-sectional study. BMC Musculoskelet Disord 2017;18:51.

34. Shigematsu H, Cheung JP, Bruzzone M, et al. Preventing fusion mass shift avoids postoperative distal curve adding-on in adolescent idiopathic scoliosis. Clin Orthop Relat Res 2017;475:1448-60.

35. Yao G, Cheung JP, Shigematsu H, et al. Characterization and predictive value of segmental curve flexibility in adolescent idiopathic scoliosis patients. Spine (Phila Pa 1976) 2017;42:1622-8. 